\title{
QUALIDADE DE MUDAS DE Murraya paniculata PRODUZIDAS EM DIFERENTES SUBSTRATOS
}

\author{
Paulo André Trazzi ${ }^{1}$, Marcos Vinicius Winckler Caldeira ${ }^{2}$, Rogério Colombi ${ }^{3}$, \\ Elzimar Oliveira Gonçalves² \\ ${ }^{1}$ Eng. Florestal, Doutorando em Engenharia Florestal, UFPR, Curitiba, PR, Brasil - patrazzi@ hotmail.com \\ ${ }^{2}$ Eng. Florestal, Dr., Depto. de Ciências Florestais e da Madeira, UFES, Alegre, ES, Brasil - caldeiramv@pq.cnpq.br; \\ elzimarog@yahoo.com.br \\ ${ }^{3}$ Eng. Florestal, Laranjeiras, ES - colombiflorestal@ hotmail.com \\ Recebido para publicação: 08/11/2010 - Aceito para publicação: 06/08/2012
}

\begin{abstract}
Resumo
Um dos aspectos mais importantes para o estabelecimento de bons povoamentos florestais é a utilização de mudas de qualidade. O substrato é um fator que exerce grande influência e por isso deve ser constituído de materiais que fornecem propriedades capazes de suprir as necessidades das mudas. $\mathrm{O}$ presente trabalho teve como objetivo avaliar a influência da utilização de lodo de esgoto e dejetos bovinos como componente de substrato na qualidade de mudas de Murraya paniculata (L.) Jack. As mudas foram produzidas em sacolas plásticas de $12 \times 18 \mathrm{~cm}$, com capacidade volumétrica de $2 \mathrm{~L}$, contendo como substrato diferentes combinações entre lodo de esgoto, dejetos bovinos e terra de subsolo. Aos 150 dias após a repicagem, foi observado que a utilização de lodo de esgoto ou dejetos bovinos na composição do substrato influenciou significativamente as características morfológicas das mudas, existindo uma tendência de crescimento das mudas em altura, diâmetro e biomassa à medida que aumenta a proporção de lodo de esgoto e dejetos bovinos. Para a produção de mudas de Murraya paniculata, a utilização de substratos que contenham quantidades maiores que $60 \%$ de dejetos bovinos ou lodo de esgoto em mistura com terra de subsolo pode ser recomendada.

Palavras-chave: Murraya paniculata; mudas; substrato; lodo de esgoto; dejetos de bovinos.
\end{abstract}

\begin{abstract}
Seedling quality of Murraya paniculata produced with different substrates. One of the most important aspects for the establishment of good forest stands is the use of high quality seedlings. The substrate is a factor that exerts great influence and therefore should be made of materials that provide properties that meet the needs of plants. This study aimed to evaluate the influence of the use of sewage sludge and cattle manure as a component of the substrate quality of seedlings of Murraya paniculata (L.) Jack. The seedlings were grown in plastic bags of $12 \times 18 \mathrm{~cm}$, with a volume capacity of $2 \mathrm{~L}$, containing as substrate, different combinations of sewage sludge, cattle manure and subsoil. At 150 days after transplantation was observed that the use of sewage sludge or cattle manure in the substrate composition significantly influenced the morphological characteristics of seedlings, there is a tendency of growth of seedlings in height, diameter and biomass with increasing of the proportion of sewage sludge and cattle manure. For the production of seedlings of Murraya paniculata, the use of substrates containing larger quantities than $60 \%$ of cattle manure or sewage sludge mixed with subsoil may be recommended.
\end{abstract}

Keywords: Murraya paniculata; plants; substrate; sewage sludge; cattle manure.

\section{INTRODUÇÃO}

Para a produção de mudas de espécies florestais para plantios comerciais, recuperação de áreas degradadas ou recomposição de florestas, se faz necessário a utilização de alternativas que visam à redução dos custos de manejo dessas espécies no viveiro. Produzir mudas resistentes, mais capacitadas a sobreviver às adversidades encontradas, é uma das possíveis opções para minimizar as perdas pós-plantio.

A necessidade de produzir mudas em viveiros, com características específicas e condições controladas, se deve ao fato de elas serem geralmente frágeis, precisando de proteção inicial e de manejos especiais, de maneira a obter-se maior uniformização de crescimento, promovendo uma rustificação, de 
forma que, após o plantio, as mudas resistam às condições adversas encontradas, sobrevivam e depois cresçam satisfatoriamente (GOMES et al., 2002).

Para a obtenção de mudas de qualidade, se torna necessária a utilização de uma boa técnica de formação de mudas, e entre os fatores importantes está o substrato. Este tem por finalidade garantir o desenvolvimento de uma planta com qualidade, em curto período de tempo e baixo custo (CUNHA et al., 2006; CALDEIRA et al., 2008). As seguintes características são consideradas fundamentais para um substrato (GONÇALVES et al., 2000): estrutura e consistência, boa porosidade e capacidade de retenção de água, isenção de substâncias tóxicas, de inóculos de doenças, de plantas invasoras, de insetos, de sais em excesso e com disponibilidade em quantidade adequada e custos economicamente viáveis.

Existem vários tipos de substratos que podem ser utilizados para produção de mudas, no entanto, na tentativa de contornar os transtornos causados pela crescente produção de lixo e resíduos, têm-se buscado estratégias de reutilização desses rejeitos, uma das quais é como componente de substrato.

Um dos produtos resultantes da estação de tratamento de esgoto (ETE) é o lodo de esgoto, que, após sofrer estabilização biológica, torna-se um resíduo que apresenta composições variáveis, no que diz respeito ao teor de matéria orgânica e disponibilidade de nutrientes (SILVA et al., 2002). Estudos com a finalidade de viabilizar sua utilização representam uma demanda atual, visto que os sistemas de produção são favorecidos pelo uso de insumos regionais e de baixo custo (AUGUSTO et al., 2003).

O lodo de esgoto é comprovadamente um excelente fornecedor de matéria orgânica, capaz de melhorar as propriedades físicas do solo (JORGE et al., 1991). No entanto, a utilização desse material no meio agrícola apresenta algumas restrições. Uma delas é a presença de metais pesados, que não apenas exercem efeito negativo sobre o crescimento das plantas como também afetam os processos bioquímicos que ocorrem no solo (HATTARI; BROADVENT, 1991).

Na composição do substrato para o crescimento de plântulas, a fonte orgânica é responsável pela retenção de umidade e fornecimento de parte dos nutrientes (CUNHA et al., 2006). Tradicionalmente, os dejetos bovinos são utilizados como fonte orgânica na composição de substratos para viveiros de mudas de café, de plantas hortícolas e de plantas arbóreas (FONSECA, 1988; SANTOS et al., 1994; ANDRADE NETO et al., 1999).

Os critérios usados na avaliação da qualidade de mudas são baseados nos valores das características morfofisiológicas do crescimento, obtidos ao final do período de produção, no viveiro, e que são peculiares de cada espécie. Entre essas características, altura da parte aérea, diâmetro de colo, relação H/D e massa seca são as mais frequentemente estudadas (CARNEIRO et al. 2007).

As características morfológicas são atributos determinados visual ou fisicamente, devendo ser ressaltado que algumas pesquisas têm sido realizadas com o intuito de mostrar que os critérios que adotam essas características são importantes para o sucesso do desempenho das mudas após o plantio (FONSECA, 2000).

Quanto à espécie florestal, murta-de-cheiro (Murraya paniculata (L.) Jack) é uma planta nacionalmente conhecida pela sua versatilidade, beleza e resistência, sendo frequentemente utilizada para a formação de cercas-vivas e na confecção de arranjos florais. A espécie cresce comumente em áreas planas, sendo nativa e normalmente encontrada na Índia, Birmânia e Malásia, além de frequentemente cultivada na Tailândia, Camboja, Vietnã e África Oriental (SHARKER et al., 2009).

Conforme o exposto acima, o objetivo desse trabalho foi avaliar a qualidade de mudas de Murraya paniculata em diferentes composições de lodo de esgoto e dejetos bovinos.

\section{MATERIAL E MÉTODOS}

O presente estudo foi realizado no Viveiro Florestal do Departamento de Ciências Florestais e da Madeira (DCFM)/CCA-UFES, Alegre, ES, sendo composto por três experimentos, totalizando 14 tratamentos. Os tratamentos foram constituídos por diferentes proporções entre terra de subsolo (TS), lodo de esgoto (LE) e dejetos bovinos (DB) (Tabela 1).

Foi utilizado o delineamento experimental inteiramente casualizado, com seis repetições por tratamento, sendo cada repetição constituída por três mudas, perfazendo 18 em cada tratamento. As médias foram comparadas pelo teste de Tukey a $5 \%$ de probabilidade.

A terra de subsolo utilizada foi um Latossolo Vermelho-Amarelo (EMBRAPA, 2006) coletado na profundidade de 20-40 cm, na Área Experimental I do CCA-UFES, Alegre, ES. 
O lodo de esgoto utilizado no experimento foi doado pela Foz do Brasil S.A. (ETE de Cachoeiro de Itapemirim, ES). Na tabela 2, observa-se que as quantidades de metais pesados presentes no lodo de esgoto são baixas, e de acordo com a resolução CONAMA 375/2006, esse material está apto para uso em ambientes agrícolas.

Tabela 1. Composição dos tratamentos (\%) presentes nos três experimentos.

Table 1. Composition of the treatments (\%) in these three experiments.

\begin{tabular}{|c|c|c|c|}
\hline Tratamentos & Terra de subsolo (TS) & Lodo de esgoto (LE) & Dejetos bovinos (DB) \\
\hline \multicolumn{4}{|c|}{ Experimento I } \\
\hline T1 & 80 & 20 & - \\
\hline $\mathrm{T} 2$ & 60 & 40 & - \\
\hline $\mathrm{T} 3$ & 40 & 60 & - \\
\hline $\mathrm{T} 4$ & 20 & 80 & - \\
\hline \multicolumn{4}{|c|}{ Experimento II } \\
\hline T5 & 80 & - & 20 \\
\hline T6 & 60 & - & 40 \\
\hline $\mathrm{T} 7$ & 40 & - & 60 \\
\hline $\mathrm{T} 8$ & 20 & - & 80 \\
\hline \multicolumn{4}{|c|}{ Experimento III } \\
\hline T9 & 20 & 20 & 60 \\
\hline T10 & 20 & 40 & 40 \\
\hline T11 & 20 & 60 & 20 \\
\hline T12 & 40 & 40 & 20 \\
\hline T13 & 40 & 20 & 40 \\
\hline T14 & 60 & 20 & 20 \\
\hline
\end{tabular}

Tabela 2. Teores $\left(\mathrm{mg}^{\mathrm{dm}} \mathrm{m}^{-3}\right)$ de metais pesados no lodo de esgoto doado pela Foz do Brasil S.A. na ETE de Cachoeiro de Itapemirim (ES).

Table 2. Content $\left(\mathrm{mg}^{-\mathrm{dm}^{-3}}\right)$ heavy metal in sewage sludge donated by SA in Foz do Brasil S.A. in the treatment plant sewer Cachoeiro de Itapemirim (ES).

\begin{tabular}{lcc}
\hline Metais & Resultados analíticos & Resolução CONAMA 375/2006 \\
\hline $\mathrm{Ar}$ & $<0,5$ & 41 \\
$\mathrm{Ba}$ & 156 & 1300 \\
$\mathrm{Cd}$ & $<0,053$ & 39 \\
$\mathrm{~Pb}$ & 29 & 300 \\
$\mathrm{Cu}$ & 98 & 1500 \\
$\mathrm{Cr}$ & 26 & 1000 \\
$\mathrm{Mb}$ & 3,5 & 50 \\
$\mathrm{Ni}$ & 11 & 420 \\
$\mathrm{Se}$ & $<0,5$ & 100 \\
\hline
\end{tabular}

Os dejetos bovinos foram obtidos do próprio CCA-UFES e passaram por processo de estabilização biológica no próprio Viveiro Florestal do DCFM, onde permaneceram depositados em local aberto durante cerca de 30 dias. $\mathrm{O}$ lodo de esgoto e os dejetos bovinos foram peneirados em malha de $3 \mathrm{~mm}$ para utilização como substrato.

Quanto aos recipientes para a produção de mudas, foram usadas sacolas plásticas com dimensões de 12,0 x 18,0 cm, com capacidade volumétrica de $2 \mathrm{~L}$ de substrato. Para o seu enchimento, utilizou-se proveta graduada para medir os volumes dos componentes a serem misturados. Os substratos formados foram homogeneizados e preenchidos, passando por uma leve compactação. As sacolas foram acondicionadas em bandejas dispostas em canteiro suspensos a $80 \mathrm{~cm}$ do solo, na casa de sombra, coberta com sombrite $(50 \%)$. 
As sementes de $M$. paniculata foram extraídas a partir de frutos coletadas aleatoriamente em árvores presentes na arborização urbana da cidade de Alegre (ES). Os frutos permaneceram em água por 24 horas, sendo despolpados em seguida, para proporcionar uma germinação mais eficiente e uniforme das sementes. Posteriormente, foi efetuada a semeadura em sementeira de areia, onde as plântulas, após atingirem altura entre cinco e sete centímetros, foram repicadas para as sacolas plásticas, já com os substratos de seus respectivos tratamentos. O tempo de permanência das mudas na casa de sombra durante o experimento foi de 150 dias.

Para avaliar a influência dos diferentes substratos na produção das mudas, as seguintes características morfológicas foram avaliadas: diâmetro de colo (D), medido na muda ao nível do substrato com uso de um paquímetro digital; altura da parte aérea $(\mathrm{H})$, determinada a partir do colo da muda até a inserção da última folha, com auxílio de uma régua graduada em milímetros; massa seca radicular (MSR), cujas raízes foram separadas da parte aérea, lavadas em peneiras, acondicionadas em sacos de papel e colocadas para secar em estufa com circulação forçada de ar a $70{ }^{\circ} \mathrm{C}$, até a obtenção de peso de matéria seca constante; massa seca da parte aérea (MSPA), separada ao nível do colo, obtida a partir do material seco em estufa, com circulação forçada de ar a $70{ }^{\circ} \mathrm{C}$, até atingir peso de matéria seca constante; massa seca total (MST), calculada através da soma da MSPA e da MSR; relação entre a altura da parte aérea e o diâmetro de colo (H/D); relação entre o peso de matéria seca da parte aérea e o peso de matéria seca do sistema radicular (MSPA/MSR). Também foi calculado o índice de qualidade de Dickson (IQD) (DICKSON et al., 1960), através da fórmula:

$$
\mathrm{IQD}=\frac{\mathrm{MST}}{\mathrm{H} / \mathrm{D}+\mathrm{MSPA} / \mathrm{MSR}}
$$

em que: MST $(\mathrm{g})=$ massa seca total; $\mathrm{H}(\mathrm{cm})=$ altura; $\mathrm{D}(\mathrm{mm})=$ diâmetro do colo; MSPA $(\mathrm{g})=$ massa seca da parte aérea; MSR $(\mathrm{g})=$ massa seca da raiz.

Amostras de terra de subsolo, lodo de esgoto e dejetos bovinos foram enviadas para o Laboratório do DCFM em Jerônimo Monteiro (ES), para análise química, de acordo com a metodologia proposta pela EMBRAPA (1997), sendo os resultados expressos na tabela 3.

Tabela 3. Análise química dos componentes dos substratos.

Table 3. Chemical analysis of the components of the substrates.

\begin{tabular}{|c|c|c|c|c|c|c|c|c|c|c|c|}
\hline \multirow{2}{*}{ Substratos } & \multirow{2}{*}{$\begin{array}{c}\mathbf{p H} \\
\mathrm{H}_{2} \mathrm{O}\end{array}$} & $\mathbf{P}$ & $\mathbf{K}$ & $\mathbf{N a}$ & $\mathbf{C a}$ & Mg & Al & H+Al & CTC & MO & $\mathbf{N}_{\text {total }}$ \\
\hline & & \multicolumn{3}{|c|}{$\left(\mathrm{mg} . \mathrm{dm}^{-3}\right)$} & \multicolumn{5}{|c|}{$\left(\mathrm{cmol}_{\mathrm{c}} \cdot \mathrm{dm}^{-3}\right)$} & \multicolumn{2}{|c|}{$\left.(\text { g.kg })^{-1}\right)$} \\
\hline Terra de subsolo & 6,9 & 17 & 21 & 5 & 2,9 & 1,0 & 0,0 & 0,7 & 4,72 & 7,0 & 0,4 \\
\hline Lodo de esgoto & 5,0 & 195 & 68 & 17 & 16,3 & 2,1 & 0,1 & 9,8 & 28,5 & 65,0 & 13,3 \\
\hline Dejetos bovinos & 8,3 & 532 & 4825 & 205 & 5,1 & 5,4 & 0,0 & 0,0 & 23,75 & 84,0 & 4,6 \\
\hline
\end{tabular}

\section{RESULTADOS E DISCUSSÃO}

A utilização de diferentes proporções de lodo de esgoto e dejetos bovinos na composição do substrato proporcionou um efeito significativo $(\mathrm{p}<0,01$ e $\mathrm{p}<0,05)$ para as características morfológicas altura, diâmetro de colo, massa seca da parte aérea e massa seca radicular nos três experimentos (Tabela 4).

Foi observada uma tendência de crescimento das mudas em altura, diâmetro de coleto e biomassa à medida que aumenta a proporção de lodo de esgoto e dejetos bovinos como componente de substrato. Isso pode ser atribuído a altos teores de $\mathrm{N}$ e P na composição desses resíduos (Tabela 3). De acordo com Novais et al. (1990), nutrientes como N e P são altamente requeridos nos estádios iniciais de desenvolvimento das mudas. Teores razoáveis de nutrientes, como $\mathrm{Ca}, \mathrm{Mg}, \mathrm{S}$, com destaque $\mathrm{N}$ e $\mathrm{P}$, são encontrados em biossólidos de origens residencial e industrial, conforme constatado por Guerrini e Trigueiro (2004), ao estudá-los na produção de mudas de Eucaliptus grandis.

De acordo com a tabela 4, observou-se que, para os três experimentos, os maiores valores para crescimento em altura e diâmetro de colo foram obtidos nos tratamentos com a maior proporção de dejetos bovinos e/ou lodo de esgoto. Gomes e Paiva (2006) afirmam que a altura, combinada com seu diâmetro de colo, constitui uma das mais importantes características morfológicas para estimar o crescimento das mudas. Para Faustino et al. (2005), os incrementos em altura estão relacionados aos acréscimos de matéria orgânica no substrato. 
Tabela 4. Valores médios de altura (H), diâmetro de colo (D), massa seca parte aérea (MSPA), massa seca radicular (MSR) de mudas de Murraya paniculata produzidas em diferentes composições de substratos.

Table 4. Average values of height $(\mathrm{H})$, basal diameter (DC), shoot dry mass (MSPA), root dry mass (MSR) in seedlings of Murraya paniculata produced in different substrate compositions.

\begin{tabular}{|c|c|c|c|c|}
\hline Substratos $^{1}$ & $\mathbf{H}(\mathbf{c m})$ & $\mathbf{D}(\mathbf{m m})$ & MSPA (g.planta ${ }^{-1}$ ) & $\operatorname{MSR}\left(\right.$ g.planta ${ }^{-1}$ ) \\
\hline \multicolumn{5}{|c|}{ Experimento I } \\
\hline $\mathrm{T} 1(80+20+0)$ & $20,9 \mathrm{c}$ & $3,17 \mathrm{c}$ & $2,42 \mathrm{c}$ & $0,61 \mathrm{c}$ \\
\hline $\mathrm{T} 2(60+40+0)$ & $24,0 \mathrm{~b}$ & $3,43 \mathrm{c}$ & $2,59 \mathrm{c}$ & $0,69 \mathrm{bc}$ \\
\hline $\mathrm{T} 3(40+60+0)$ & $25,3 \mathrm{~b}$ & $3,77 \mathrm{~b}$ & $3,32 \mathrm{~b}$ & $0,78 \mathrm{~b}$ \\
\hline $\mathrm{T} 4(20+80+0)$ & $31,6 \mathrm{a}$ & $4,28 \mathrm{a}$ & $4,52 \mathrm{a}$ & $0,95 \mathrm{a}$ \\
\hline $\mathrm{F}$ & $* *$ & $* *$ & $* *$ & $* *$ \\
\hline $\mathrm{CV} \%$ & 6,46 & 4,72 & 6,90 & 10,67 \\
\hline \multicolumn{5}{|c|}{ Experimento II } \\
\hline $\mathrm{T} 5(80+0+20)$ & $9,1 \mathrm{~b}$ & $2,28 \mathrm{~b}$ & $0,58 \mathrm{c}$ & $0,21 \mathrm{~b}$ \\
\hline $\mathrm{T} 6(60+0+40)$ & $11,0 \mathrm{~b}$ & $2,46 \mathrm{ab}$ & $0,76 \mathrm{c}$ & $0,25 \mathrm{~b}$ \\
\hline $\mathrm{T} 7(40+0+60)$ & $14,9 \mathrm{a}$ & $2,89 \mathrm{a}$ & $1,40 \mathrm{~b}$ & $0,47 \mathrm{a}$ \\
\hline $\mathrm{T} 8(20+0+80)$ & $17,1 \mathrm{a}$ & $2,96 \mathrm{a}$ & $1,96 \mathrm{a}$ & $0,57 \mathrm{a}$ \\
\hline $\mathrm{F}$ & $* *$ & $* *$ & $* *$ & $* *$ \\
\hline $\mathrm{CV} \%$ & 11,00 & 11,58 & 16,89 & 20,99 \\
\hline \multicolumn{5}{|c|}{ Experimento III } \\
\hline T9 $(20+20+60)$ & $28,7 \mathrm{ab}$ & $4,0 \mathrm{ab}$ & $4,08 \mathrm{abc}$ & $0,81 \mathrm{c}$ \\
\hline $\mathrm{T} 10(20+40+40)$ & $31,7 \mathrm{a}$ & $4,3 \mathrm{a}$ & $4,86 \mathrm{a}$ & $1,03 \mathrm{ab}$ \\
\hline $\mathrm{T} 11(20+60+20)$ & $31,5 \mathrm{a}$ & $4,3 \mathrm{a}$ & $4,42 \mathrm{abc}$ & $0,86 \mathrm{bc}$ \\
\hline $\mathrm{T} 12(40+40+20)$ & $31,2 \mathrm{a}$ & $4,3 \mathrm{a}$ & $4,69 \mathrm{ab}$ & $1,07 \mathrm{a}$ \\
\hline $\mathrm{T} 13(40+20+40)$ & $25,5 \mathrm{~b}$ & $3,5 \mathrm{~b}$ & $3,22 \mathrm{~d}$ & $0,76 \mathrm{c}$ \\
\hline $\mathrm{T} 14(60+20+20)$ & $29,9 \mathrm{ab}$ & $4,2 \mathrm{ab}$ & $4,03 \mathrm{c}$ & $0,95 \mathrm{abc}$ \\
\hline $\mathrm{F}$ & $*$ & $*$ & $* *$ & $* *$ \\
\hline $\mathrm{CV} \%$ & 9,60 & 9,03 & 7,34 & 11,02 \\
\hline
\end{tabular}

${ }^{1}$ Terra de subsolo + lodo de esgoto + dejetos bovinos; *: significativo $(\mathrm{p}<0,05)$; **: significativo (p <0,01). Médias seguidas da mesma letra, na coluna, não diferem estatisticamente entre si pelo teste Tukey $(\mathrm{p}>0,05)$.

Em estudo com Schinus terebinthifolius, Archontophoenix alexandrae e Archontophoenix cunninghamiana, Caldeira et al. (2007) observaram que o substrato padrão (50\% de dejetos bovinos, 25\% de casca de arroz e $25 \%$ de argila) apresentou resultados semelhantes para o crescimento em altura, quando comparados aos tratamentos à base de resíduos da indústria de algodão, para as três espécies. Para o diâmetro de colo, o substrato padrão promoveu o menor crescimento para Schinus terebinthifolius, sendo que a maior média para essa característica foi obtida no tratamento com $75 \%$ de resíduos da indústria de algodão. Para as espécies do gênero Archontophoenix, não houve diferenças significativas entre os tratamentos para o crescimento em diâmetro de colo.

As características morfológicas MSPA e MSR apresentaram maiores valores médios para as mudas produzidas nos substratos com maiores proporções de matéria orgânica, seja com adição de lodo de esgoto ou de dejetos bovinos (Tabela 4). A disponibilidade de nutrientes presentes nos resíduos (Tabela 3), principalmente $\mathrm{P}$, influenciou significativamente o crescimento inicial das mudas de $M$. paniculata. Da mesma forma, Santos et al. (2008), em estudo com sete espécies arbóreas nativas, verificaram que, ao se elevar o fornecimento de $\mathrm{P}$, ocorreram aumentos na produção de matéria seca da parte aérea para as espécies estudadas. Ao contrário, um estudo realizado por Cunha et al. (2006) com mudas de Acacia mangium e Acacia auriculiformis indicou que as mudas produzidas com menor quantidade de lodo de esgoto foram as que tiveram maior produção de biomassa de raízes.

De maneira geral, observa-se que, no experimento I, o tratamento T4 (20TS + 80LE) apresentou as maiores médias de H, D, MSPA e MSR, diferenciando-se estatisticamente dos demais tratamentos. O 
mesmo ocorreu no experimento II, cujo tratamento T8 (20TS + 80DB) se diferenciou dos demais tratamentos, apresentando a maior média para essas variáveis. Similarmente, no experimento III, as maiores médias foram obtidas nos tratamentos T10, T11 e T12, com maior quantidade de matéria orgânica, o que possibilitou obter mudas com maior crescimento em altura, diâmetro e produção de biomassa.

Para a relação entre a altura da parte aérea e o diâmetro de colo, relação entre o peso de matéria seca da parte aérea e da matéria seca do sistema radicular, massa seca total e índice de qualidade de Dickson, a utilização de lodo de esgoto e dejetos bovinos como componente de substrato apresentou efeito significativo ( $\mathrm{p}<0,01$ e $\mathrm{p}<0,05)$ para a maioria das características morfológicas analisadas nos três experimentos. A relação H/D não foi significativa $(p>0,05)$ nos experimentos I e III e a relação MSPA/MSR no experimento I e II também não apresentou resultados significativos ( $p>0,05)$, conforme tabela 5 .

Tabela 5. Valores médios da relação altura/diâmetro (H/D), relação massa seca da parte aérea e massa seca radicular (MSPA/MSR), massa seca total (MST) e índice de qualidade de Dickson (IQD) em mudas de Murraya paniculata produzidas em diferentes composições de substratos.

Table 5. Average values of ratio height/diameter (RHD), ratio dry mass shoot/root (MSPA/MSR), total dry mass (MST) and Dickson quality index (IQD) in seedlings of Murraya paniculata produced in different substrate compositions.

\begin{tabular}{|c|c|c|c|c|}
\hline Substratos $^{1}$ & H/D & MSPA/MSR & MST (g.planta ${ }^{-1}$ ) & IQD \\
\hline \multicolumn{5}{|c|}{ Experimento I } \\
\hline $\mathrm{T} 1(80+20+0)$ & 6,6 & 4,0 & $3,03 \mathrm{c}$ & $0,29 \mathrm{c}$ \\
\hline $\mathrm{T} 2(60+40+0)$ & 7,0 & 3,7 & $3,28 \mathrm{c}$ & $0,30 \mathrm{c}$ \\
\hline $\mathrm{T} 3(40+60+0)$ & 6,7 & 4,2 & $4,10 \mathrm{~b}$ & $0,38 \mathrm{~b}$ \\
\hline $\mathrm{T} 4(20+80+0)$ & 7,4 & 4,8 & $5,47 \mathrm{a}$ & $0,45 \mathrm{a}$ \\
\hline $\mathrm{F}$ & ns & ns & $* *$ & $* *$ \\
\hline $\mathrm{CV} \%$ & 7,32 & 14,54 & 4,85 & 5,69 \\
\hline \multicolumn{5}{|c|}{ Experimento II } \\
\hline $\mathrm{T} 5(80+0+20)$ & $3,9 \mathrm{c}$ & 2,8 & $0,79 \mathrm{c}$ & $0,11 \mathrm{~b}$ \\
\hline $\mathrm{T} 6(60+0+40)$ & $4,5 \mathrm{bc}$ & 3,0 & $1,01 \mathrm{c}$ & $0,13 \mathrm{~b}$ \\
\hline $\mathrm{T} 7(40+0+60)$ & $5,1 \mathrm{ab}$ & 3,1 & $1,88 \mathrm{~b}$ & $0,22 \mathrm{a}$ \\
\hline $\mathrm{T} 8(20+0+80)$ & $5,8 \mathrm{a}$ & 3,5 & $2,54 \mathrm{a}$ & $0,27 \mathrm{a}$ \\
\hline $\mathrm{F}$ & $* *$ & $\mathrm{~ns}$ & $* *$ & $* *$ \\
\hline $\mathrm{CV} \%$ & 8,34 & 25,79 & 14,44 & 14,72 \\
\hline \multicolumn{5}{|c|}{ Experimento III } \\
\hline T9 $(20+20+60)$ & 7,1 & $5,1 \mathrm{a}$ & $4,90 \mathrm{~b}$ & $0,40 \mathrm{bc}$ \\
\hline $\mathrm{T} 10(20+40+40)$ & 7,2 & $4,7 \mathrm{a}$ & $5,90 \mathrm{a}$ & $0,49 \mathrm{a}$ \\
\hline $\mathrm{T} 11(20+60+20)$ & 7,3 & $5,1 \mathrm{a}$ & $5,28 \mathrm{ab}$ & $0,42 \mathrm{abc}$ \\
\hline $\mathrm{T} 12(40+40+20)$ & 7,2 & $4,3 \mathrm{a}$ & $5,76 \mathrm{a}$ & 0,49 a \\
\hline $\mathrm{T} 13(40+20+40)$ & 7,3 & $4,2 \mathrm{a}$ & $3,98 \mathrm{c}$ & $0,34 \mathrm{c}$ \\
\hline $\mathrm{T} 14(60+20+20)$ & 7,1 & $4,2 \mathrm{a}$ & $4,98 \mathrm{~b}$ & $0,43 \mathrm{ab}$ \\
\hline $\mathrm{F}$ & $\mathrm{ns}$ & $*$ & $* *$ & $* *$ \\
\hline $\mathrm{CV} \%$ & 9,51 & 12,15 & 6,85 & 9,74 \\
\hline
\end{tabular}

Para as relações H/D e MSPA/MSR, só houve diferenças significativas no experimento II, cuja maior proporção de dejetos bovinos (T7 e T8) apresentou as maiores médias da relação H/D. As relações H/D e MSPA/MSR são bons indicativos para se estimar a qualidade das mudas (PARVIAINEM, 1981; CARNEIRO, 1995; GOMES; PAIVA, 2006). Trigueiro e Guerrini (2003), avaliando a utilização de lodo de esgoto na produção de mudas de eucalipto, constataram que os maiores valores das relações H/D e MSPA/MSR foram obtidos com a utilização de lodo de esgoto e casca de arroz carbonizada na proporção de 50:50 e 40:60, respectivamente.

A massa seca total foi maior nos tratamentos com a maior quantidade de lodo de esgoto e/ou dejetos bovinos nos três experimentos (T4, T8, T10, T11 e T12). É possível inferir que o incremento de 
biomassa das mudas de $M$. paniculata ocorreu em função da melhoria das condições de crescimento, ou seja, incorporação de matéria orgânica no substrato, e possibilitou maior disponibilidade de nutrientes, decorrentes da própria composição do lodo de esgoto e de dejetos bovinos (Tabela 3). Nesse sentido, Nóbrega et al. (2007), avaliando o crescimento de mudas de Schinus terebynthifolius submetidas a diferentes quantidades de solo e lodo de esgoto $(100: 0 ; 80: 20 ; 60: 40 ; 40: 60 ; 20: 80)$ sem adubação mineral, constataram que o lodo de esgoto melhorou a fertilidade do substrato, o que proporcionou aumento na biomassa total. Sarcinelli et al. (2004), avaliando a produção de biomassa de mudas de Acacia holosericea submetidas a diferentes soluções nutritivas com exclusão de macronutrientes, constataram que as maiores reduções na produção de biomassa total foram verificadas para as mudas submetidas aos tratamentos sem $\mathrm{N}$ e Mg. Sendo assim, é possível considerar que esses elementos são fundamentais para o incremento da MST de Acacia holosericea. No presente estudo, as mudas de $M$. paniculata tendem a apresentar comportamento similar, uma vez que os maiores valores de biomassa total foram obtidos nos tratamentos com a maior quantidade de lodo de esgoto e dejetos bovinos, cujos teores de N e Mg são altos (Tabela 3). A MST tem sido considerada um dos melhores fatores para caracterizar a qualidade de mudas, apresentando, porém, o inconveniente de não ser viável a sua determinação em muitos viveiros, principalmente por envolver a destruição completa da muda e a utilização de estufas (AZEVEDO, 2003).

Os tratamentos que apresentaram maior IQD foram aqueles com as maiores proporções de lodo de esgoto e dejetos bovinos como componentes dos substratos (T4, T8, T10, T11 e T12). O IQD é apontado como bom indicador de qualidade de mudas, porque são utilizados para seu cálculo a robustez (relação H/DC) e o equilíbrio da distribuição da biomassa (relação MSPA/MSR) (FONSECA, 2000; CALDEIRA et al., 2005; CALDEIRA et al., 2007; TRAZZI, 2011). Sendo assim, esses tratamentos são mais indicados para a produção de mudas de $M$. paniculata, pois quanto maior o valor desse índice, melhor o padrão de qualidade das mudas (GOMES, 2001).

É possível constatar que vários estudos na literatura mostram que o IQD é uma característica variável (CALDEIRA et al., 2000a; CALDEIRA et al., 2000b; CALDEIRA et al., 2007; CALDEIRA et al., 2005; CALDEIRA et al., 2008a; CALDEIRA et al., 2008b; SAIDELLES et al., 2009; TRAZZI et al., 2010; KRATZ, 2011; TRAZZI, 2011). Nesse sentido, pode-se concluir que esse índice pode variar em função da espécie, do manejo das mudas no viveiro, do tipo e proporção do substrato, do volume do recipiente e, principalmente, de acordo com a idade em que a muda foi avaliada.

De modo geral, os melhores resultados, para todas as características avaliadas neste estudo, foram obtidos com a maior quantidade de lodo de esgoto e dejetos bovinos nos três experimentos. Isso se deve ao fato de esses resíduos apresentarem bom teor de alguns nutrientes e matéria orgânica (Tabela 3). Segundo Malavolta et al. (2002), alguns adubos orgânicos são empregados em doses elevadas, pois são pobres nos elementos nutritivos como nitrogênio, fósforo e potássio. Outros adubos, mais concentrados, comportam-se de modo mais semelhante ao dos adubos minerais, funcionando como fonte de nitrogênio, fósforo, potássio e outros elementos. Para Melo (2000), a matéria orgânica, além de se constituir em um dos principais, senão o principal, componente da CTC, durante o processo de mineralização, libera nutrientes para a nutrição das plantas.

\section{CONCLUSÕES}

- Mudas de M. paniculata de melhor qualidade foram obtidas a partir do uso de lodo de esgoto e de dejetos bovinos como componente de substrato.

- A utilização de substratos que contenham quantidades maiores que $60 \%$ de dejetos bovinos ou lodo de esgoto em mistura com terra de subsolo pode ser recomendada para a produção de mudas de $M$. paniculata.

- O lodo de esgoto e os dejetos bovinos podem se tornar alternativas como componentes de substrato na produção de mudas de espécies florestais. Além do benefício ambiental, proporcionam economia de fertilizantes.

\section{AGRADECIMENTOS}

Os autores agradecem à CAPES, pela concessão de bolsa de doutorado, e ao CNPq, pela bolsa de produtividade em pesquisa. 


\section{REFERÊNCIAS}

ANDRADE NETO, A.; MENDES, A. N. G.; GUIMARÃES, P. T. G. Avaliação de substratos alternativos e tipo de adubação para a produção de mudas de cafeeiro em tubetes. Ciência e Agrotecnologia, v. 23, n. 2, p. 270 - 280, 1999.

AUGUSTO, D. C. C.; GERRINI, I. A.; ENGEL, V. L.; ROUSSEAU, G. X. Utilização de esgotos domésticos tratados através de um sistema biológico na produção de mudas de Cróton floribundus Spreng. (capixingui) e Copaifera langsdorffii Desf. (copaíba). Árvore, v. 27, p. 335 - 342, 2003.

AZEVEDO, M. I. R. Qualidade de mudas de cedro-rosa (Cedrela fissilis Vell.) e de ipê-amarelo (Tabebuia serratifolia (Vahl) Nich.) produzidas em diferentes substratos e tubetes. 90f. Dissertação (Mestrado em Ciência Florestal) - Universidade Federal de Viçosa, Viçosa, 2003.

CALDEIRA, M. V. W.; BLUM, H.; BALBINOT, R.; LOMBARDI, K. C. Uso do resíduo do algodão no substrato para produção de mudas florestais. Revista Acadêmica: Ciências Agrárias e Ambientais, v. 6, p. 191 - 202. 2008a.

CALDEIRA, M. V. W.; MARCOLIN, M.; MORAES, E.; SCHAADT, S. S. Influência do resíduo da indústria do algodão na formulação de substrato para produção de mudas de Schinus terebinthifolius Raddi, Archontophoenix alexandrae Wendl. et Drude e Archontophoenix cunninghamiana Wendl. et Drude. Ambiência, Guarapuava, v. 3, p. 1 - 8, 2007.

CALDEIRA, M. V. W.; MARCOLIN, M.; MORAES, E.; SCHAADT, S. S. Influência do resíduo da indústria do algodão na formulação de substrato para produção de Schinus terebinthifolius Raddi, Archontophoenix alexandrae Wendl. et Drude e Archontophoenix cunninghamiana Wendl. et Drude. Ambiência, v. 3, p. 311 - 323, 2007.

CALDEIRA, M. V. W.; ROSA, G. N. da; FENILLI, T. A. B.; HARBS, R. M. P. Composto orgânico na produção de mudas de aroeira-vermelha. Scientia Agraria, v. 9, p. 27 - 33. 2008b.

CALDEIRA, M. V. W.; SCHUMACHER, M. V.; BARICHELLO, L. R.; VOGET, H. L. M.; OLIVEIRA, L. S. Crescimento de mudas de Eucalyptus saligna Smith em função de diferentes doses de vermicomposto. Floresta, Curitiba, v. 28, n. 1/2, p. 19 - 30, 2000a.

CALDEIRA, M. V. W.; SCHUMACHER, M. V.; TEDESCO, N. Crescimento de mudas de Acacia mearnsii em função de diferentes doses de vermicomposto. Scientia Forestalis, Piracicaba, n. 57, p. 161 $170,2000 \mathrm{~b}$.

CALDEIRA, M. V. W.; SPATHELF, P.; BARICHELLO, L. R.; VOGEL, H. L. M.; SCHUMACHER, M. V. Effect of different doses of vermicompost on the growth of Apuleia leiocarpa (Vog) Macbr. seedlings. Revista Acadêmica: Ciências Agrárias e Ambientais, v. 3, p. 11 - 17, 2005.

CALDEIRA, M. V. W.; ROSA, G. N. da; FENILLI, T. A. B.; HARBS, R. M. P. Composto orgânico na produção de mudas de aroeira-vermelha. Scientia Agraria, v. 9, p. 27 - 33, 2008.

CARNEIRO, J. G. A. Produção e controle de qualidade de mudas florestais. Curitiba: UFPR/FUPEF/UENF, 1995. $451 \mathrm{p}$.

CARneIRO, J. G. A.; BARROSO, D. G.; SOARES, L. M. S. Crescimento de mudas em raiz nua de Pinus taeda, L., sob cinco espaçamentos no viveiro e seu desempenho no campo. Revista Brasileira de Agrociência, v. 13, p. 305 - 310. 2007.

CUNHA, A. M.; CUNHA, G. M.; SAMENTO, R. A.; CUNHA, G. M.; AMARAL, J. F. T. Efeito de diferentes substratos sobre o desenvolvimento de mudas de Acacia sp. Revista Árvore, v. 30, p. 207 214, 2006.

DICKSON, A.; LEAF, A.; HOSNER, J. F. Quality appraisal of white spruce and white pine seedling stock in nurseries. Forestry chronicle, v. 36, p. 10 - 13, 1960. 
EMPRESA BRASILEIRA DE PESQUISA AGROPECUÁRIA (EMBRAPA). Manual de análises químicas de solos, plantas e fertilidade. Rio de Janeiro: Embrapa Solos, 1997. 370 p.

$306 \mathrm{p}$.

Sistema Brasileiro de Classificação de Solos. 2. ed. Rio de Janeiro: EMBRAPA-SPI, 2006.

FAUSTINO, R.; KATO, M. T.; FLORÊNCIO, L.; GAVAZZA, S. Lodo de esgoto como substrato para produção de mudas de Senna siamea Lam. Revista Brasileira de Engenharia Agrícola e Ambiental, v. 9 , p. $278-282,2005$.

FONSECA, E. P. Efeito de diferentes substratos na produção de mudas de Eucalyptus grandis W. Hill ex Maiden em "Win-strip”. 81 f. Dissertação (Mestrado em Ciência Florestal) - Universidade Federal de Viçosa, Viçosa, 1988.

Padrão de qualidade de mudas de Trema micrantha (L.) Blume., Cedrela fissilis Vell. e Aspidosperma polyneuron Müll. Arg. produzidas sob diferentes períodos de sombreamento. 113f. Tese (Doutorado em Agronomia) - Universidade Estadual Paulista, Jaboticabal, 2000.

GOMES, J. M. Parâmetros morfológicos na avaliação da qualidade de mudas de Eucalyptus grandis, produzidas em diferentes tamanhos de tubete e de dosagens de N-P-K. 166f. Tese (Doutorado em Ciência Florestal) - Universidade Federal de Viçosa, Viçosa, 2001.

GOMES, J. M.; COUTO, L.; LEITE, H. G.; XAVIER, A.; GARCIA, S. L. R. Parâmetros morfológicos na avaliação da qualidade de mudas de Eucalyptus grandis. Árvore, v. 26, n. 6, p. 655 - 664, 2002.

GOMES, J. M.; PAIVA, H. N. de. Viveiros florestais (propagação assexuada). 3. ed. Viçosa: UFV, 2006.

GONÇALVES, J. L. M.; SANTARELLI, E. G.; MORAES NETO, S. P.; MANARA, M. P. Produção de mudas de espécies nativas: substrato, nutrição, sombreamento e fertilização. IN: GONÇALVES, J. L. M.; BENEDETTI, V. (eds.). Nutrição e fertilização florestal. Piracicaba, SP: IPEF, 2000, p. 427.

GUERRINI, I. A.; TRIGUEIRO, R. M. Atributos físicos e químicos de substratos compostos por biossólidos e casca de arroz carbonizada. Revista Brasileira de Ciência do Solo, v. 28, n. 6, p. 1069 1076, 2004.

HATTARI, F. H.; BROADVENT, F. E. Influence of trace metals on some soil nitrogen transformations. Journal of Environmental Quality, v. 11, p. 1 - 4, 1991.

JORGE, J. A; CAMARGO, O. A.; VALADARES, J. M. A. S. Condições físicas de um Latossolo Vermelho-escuro quatro anos após aplicação de lodo de esgoto e calcário. Revista Brasileira de Ciência do Solo, v. 15, p. 237 - 240, 1991.

KRATZ, D. Substratos renováveis na produção de mudas de Eucalyptus benthamii Maiden et Cambage e Mimosa scabrella Benth. 121f. Dissertação (Mestrado em Engenharia Florestal) Universidade Federal do Paraná, Curitiba, 2011.

MALAVOlTA, E.; GOMES, P. F.; ALCARDE, J. C. Adubos e adubações. São Paulo: Nobel, 2002. 200 p.

MELO, W. J.; MARQUES, M. O. Potencial do lodo de esgoto como fonte de nutrientes para as plantas. In: BETTIOL, W \& CAMARGO, O. A. (eds.). Impacto ambiental do uso agrícola do lodo de esgoto. Jaguariúna: Embrapa Meio Ambiente, 2000. p. 45 - 67.

NÓBREGA, R. S. A.; BOAS, R. C. V.; NÓBREGA, J. C. A.; PAULA, A. M. de; MOREIRA, F. M. S. Utilização de biossólido no crescimento inicial de mudas de aroeira (Schinus terebinthifolius Raddi). Árvore, v. 31, p. 239 - 246, 2007.

NOVAIS, R. F.; BARROS, N. F.; NEVES, J. C. L. Nutrição mineral do eucalipto. In: BARROS, N. F; NOVAIS, R. F. (eds.). Relação solo-eucalipto. Viçosa: Editora Folha de Viçosa, 1990. p. 25 - 98.

PARVIAINEM, J. V. Qualidade e avaliação de mudas florestais. In: Seminário de sementes e viveiros florestais, Curitiba. Anais... Curitiba: FUPEF, p. 59 - 90, 1981. 
SAIDELLES, F. L. F.; CALDEIRA, M. V. W.; SCHIRMER, W. N.; SPERANDIO, H. V. Casca de arroz carbonizada como substrato para produção de mudas de tamboril-da-mata e garapeira. Semina: Ciências Agrárias, v. 30, p. 1173 - 1186. 2009.

SANTOS, J. Z. L.; RESENDE, A. V.; NETO, A. E. F.; CORTE, E. F. Crescimento, acúmulo de fósforo e frações fosfatadas em mudas de sete espécies arbóreas nativas. Árvore, v. 32, n. 5, p. 799 - 807, 2008.

SANTOS, L. P.; CARVAlHO, M. M.; CARVALHO, J. G. Efeitos de doses de nitrato de potássio e esterco de curral na composição do substrato para a formação de cafeeiro. Ciência e Prática, v. 18, n. 1, p. $42-48,1994$.

SARCINELLI, T. S.; RIBEIRO JÚNIOR, E. S.; DIAS, L. E.; LYNCH, L. de S. Sintomas de deficiência nutricional em mudas de Acacia holosericea em resposta à omissão de macronutrientes. Árvore, v. 28, p. $173-181,2004$.

SHARKER, S. Md.; SHAHID, I. J.; HASANUZZAMAN, Md. Antinociceptive and bioactivity of leaves of Murraya paniculata (L.) Jack, Rutaceae. Revista Brasileira de Farmacognosia, João Pessoa, v. 19, n. 3, p. $746-748,2009$.

SILVA, J. E.; RESCK, D. V. S.; SHARMA, R. D. Alternativa agronômica para o biossólido produzido no Distrito Federal. II - Aspectos qualitativos, econômicos e práticos de seu uso. Revista Brasileira de Ciência do Solo, v. 26, p. 497 - 503, 2002.

TRAZZI, P. A. Substratos renováveis na produção de mudas de Tectona grandis Linn F. 84f. Dissertação (Mestrado em Ciências Florestais) - Universidade Federal do Espírito Santo, Alegre, 2011.

TRAZZI, P. A.; CALDEIRA, M. V. W.; COLOMBI, R. Avaliação de mudas de Tecoma stans utilizando biossólido e resíduo orgânico. Revista de Agricultura, Piracicaba, v. 85, p. 218 - 226. 2010.

TRIGUEIRO, R. M.; GUERRINI, I. A. Uso de biossólido como substrato para produção de mudas de eucalipto. Sciencia Forestalis, Piracicaba, v. 64, p. 150 - 162, 2003. 